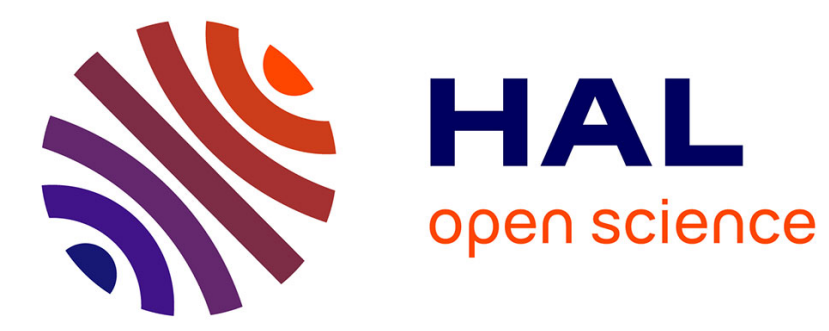

\title{
Grande vitesse ferroviaire et développement économique local: une revue de la littérature
}

Corinne Blanquart, Ligdwine Vandenbossche

\section{To cite this version:}

Corinne Blanquart, Ligdwine Vandenbossche. Grande vitesse ferroviaire et développement économique local: une revue de la littérature. colloque de l'ASRDLF : Identité, Qualité et Compétitivité Territoriale - Développement économique et cohésion dans les territoires alpins, Sep 2010, Aoste, Italie. 23p. hal-00615186

\section{HAL Id: hal-00615186 https://hal.science/hal-00615186}

Submitted on 18 Aug 2011

HAL is a multi-disciplinary open access archive for the deposit and dissemination of scientific research documents, whether they are published or not. The documents may come from teaching and research institutions in France or abroad, or from public or private research centers.
L'archive ouverte pluridisciplinaire HAL, est destinée au dépôt et à la diffusion de documents scientifiques de niveau recherche, publiés ou non, émanant des établissements d'enseignement et de recherche français ou étrangers, des laboratoires publics ou privés. 
Association de Science Régionale De Langue Française

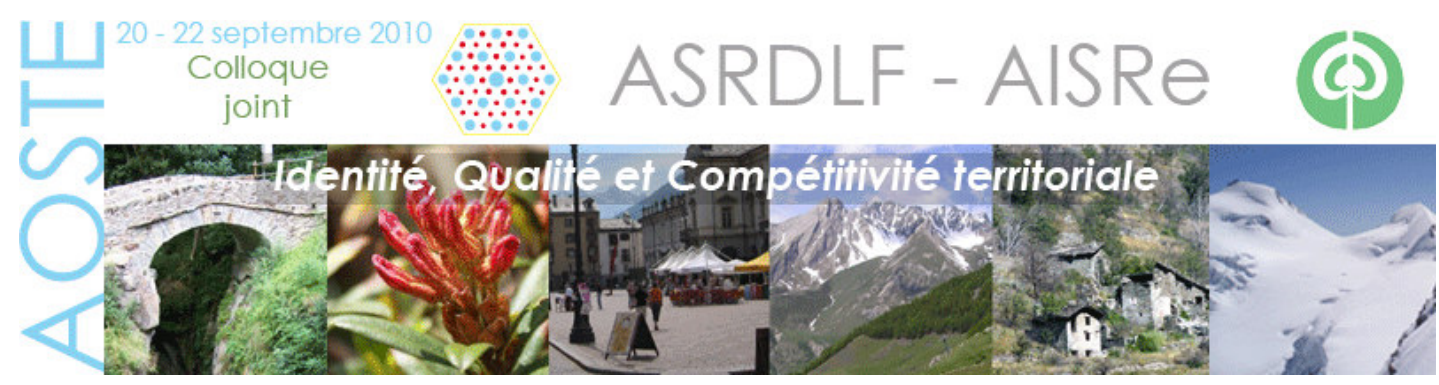

\title{
Grande vitesse ferroviaire et développement économique local : une revue de la littérature
}

\section{Bazin Sylvie, Beckerich Christophe, Delaplace Marie}

Université de Reims Champagne-Ardenne

Equipe OMI E.A. 2065

57 bis, rue Pierre Taittinger

F-51096 Reims cedex

marie.delaplace@univ-reims.fr

sylvie.bazin@univ-reims.fr

christophe.beckerich@univ-reims.fr

\author{
Blanquart Corinne, Vandenbossche Lidwine \\ INRETS-SPLOTT \\ Lille - Villeneuve D'Ascq \\ 20, Rue Elisée Reclus, BP 317 \\ F-59666 Villeneuve D'Ascq Cedex \\ corinne.blanquart@terre.inrets.fr
}

\section{Résumé :}

Des projets de lignes à grande vitesse existent dans de nombreux pays. Les acteurs économiques des territoires desservis ou qui le seront, ont eu ou ont de multiples attentes concernant cette desserte qui modifie l'accessibilité de leurs territoires: dynamisme économique, arrivée de nouvelles entreprises et populations, développement du tourisme, ou de projets immobiliers résidentiels et de bureaux autour des gares, etc. La permanence de ces attentes, alors que le mythe des effets structurants des infrastructures de transport a depuis longtemps été remis en question dans la littérature académique, est à l'origine de notre questionnement. L'objectif de cette communication est de présenter une revue de la littérature académique mais également non académique relative aux effets de la grande vitesse ferroviaire sur le développement local afin d'identifier les raisons de cette déconnexion entre les travaux académiques et les discours politiques.

\section{Mots clés :}

Infrastructure de transport, ligne à grande vitesse, développement économique local, effets structurants

Classification : R41, R42 


\section{GRANDE VITESSE FERROVIAIRE ET DEVELOPPEMENT ECONOMIQUE LOCAL : UNE REVUE DE LA LITTERATURE}

\section{INTRODUCTION}

De nombreux projets de train à grande vitesse existent au niveau international. En 2009, on comptait $10739 \mathrm{~km}$ de lignes à grande vitesse dans le monde; $13469 \mathrm{~km}$ étaient en construction et $17579 \mathrm{~km}$ étaient planifiées d'ici à 2025 et ce sur les quatre continents. A la seule échelle de l'Europe, en 2009, $5821 \mathrm{~km}$ de ligne existaient, $3256 \mathrm{~km}$ étaient en construction et pour 2025, 8501 km étaient planifiés (BARRON, 2009).

Ces projets s'inscrivent désormais dans un contexte de développement durable, et leur ambition est aussi de limiter, voire de contrer, la croissance continue du mode routier pour le déplacement des personnes. Toutefois, cette ambition de mobilité d'abord, et à l'heure actuelle de mobilité durable, s'accompagne de nombreuses autres attentes.

Les acteurs économiques des territoires qui ont été desservis ou qui le seront, ont eu ou ont en effet de nombreuses attentes concernant cette desserte qui modifie l'accessibilité de leurs territoires. Ils en espèrent de nombreux effets positifs : dynamisme économique, arrivée de nouvelles entreprises, développement du tourisme, arrivée de nouvelles populations, développement de projets immobiliers résidentiels et de bureaux autour des gares, etc. Ils anticipent également parfois des effets négatifs en termes de décroissance démographique des territoires non desservis, de ville dortoir, etc...

Ces attentes et ces craintes, que l'on retrouve dans la littérature non académique réalisée en réponse à des commandes des acteurs locaux, régionaux ou nationaux, posent d'autant plus question qu'elles semblent en décalage avec les conclusions de la littérature dite académique, publiée dans des revues scientifiques et/ou discutée dans les milieux universitaires.

Ces attentes et ces craintes ne semblent en effet pas toujours fondées, le mythe des effets structurants des infrastructures de transport ayant été depuis longtemps remis en question. Les travaux académiques ont depuis longtemps montré qu'il n'existait aucune causalité linéaire entre infrastructures de transport et transformations sociales, spatiales et économiques. De nombreuses recherches académiques montrent qu'aucun effet systématique en termes de dynamique économique ne résulte de la seule présence d'une infrastructure de transport. (BAZIN, BECKERICH, DELAPLACE et MASSON, 2006a; BLANQUART, DELAPLACE, 2009, BONNAFOUS ET PLASSARD, 1974, OFFNER, 1993, PLASSARD, 2003, 1977, VICKERMAN, 1991). Pour autant, dans certains cas, de nouvelles dynamiques émergent. Ces infrastructures de transport contribuent donc parfois à de nouvelles dynamiques, même si elles seules ne suffisent pas (TROIN, 1997). Ces dynamiques localisées, dont les medias se font l'écho, contribuent alors à perpétuer le mythe selon lequel une desserte à grande vitesse contribue à une dynamique économique locale, notamment parmi les élus locaux.

Par ailleurs, les travaux académiques, en insistant sur la complexité des phénomènes de développement, ont souligné la nécessité de stratégies d'acteurs venant accompagner la nouvelle infrastructure. Une interprétation de ces travaux laisserait donc à penser que sous réserve d'une mobilisation suffisante des acteurs, des effets seraient possibles. Si l'effet direct n'existe plus, l'effet n'a pas disparu pour autant et devient conditionné.

L'objectif de cette communication est de présenter une revue de la littérature académique mais aussi non académique de façon à éclairer et expliquer ce décalage persistant entre les connaissances académiques et non académiques. 
Nous montrerons que l'écart n'est finalement pas si grand entre les deux types de littérature. Au-delà de l'évidente justification des choix d'investissement qui incite les acteurs des territoires à une vision optimiste de l'impact des dépenses publiques, il nous semble que les effets des infrastructures sont loin d'être une question définitivement classée dans la littérature académique. Une revue de la littérature relative aux dessertes à grande vitesse nous permettra de montrer que la littérature académique continue d'identifier des effets positifs même si les travaux dépassent le plus souvent la causalité linéaire entre l'offre de services à grande vitesse et l'apparition des effets. Ce faisant, elle contribue indirectement à ce que le mythe des effets des infrastructures perdure. Ainsi dans une première partie, nous mettrons en lumière la relative proximité des attentes des acteurs des territoires avec la littérature académique : le caractère plus général de la littérature non académique, de par sa nature et sa finalité, nous semble constituer une première explication de la pérennité du mythe. En outre, nous montrerons dans une deuxième partie que si les travaux académiques identifient des conditions d'apparition des effets extrêmement diverses et souvent également plus précises, qui limitent les possibilités réelles d'effets, seules certaines d'entre elles sont évoquées dans la littérature non académique : les acteurs des territoires omettent ainsi le caractère largement contextualisé des effets et des conditions.

\section{LES ENSEIGNEMENTS D'UNE CONFRONTATION ENTRE LA LITTERATURE ACADEMIQUE ET NON ACADEMIQUE RELATIVE AUX EFFETS DES DESSERTES A GRANDE VITESSE}

L'enjeu de l'article est de sortir d'une vision réductrice opposant de façon dichotomique une littérature académique porteuse d'une position claire par rapport à la question des effets des infrastructures à grande vitesse, et une littérature non académique moins tranchée et laissant subsister des attentes, si ce n'est des espoirs, en lien avec les projets de dessertes à grande vitesse.

Une revue de ces deux littératures doit ainsi nous permettre de mettre en évidence ou de nuancer ce décalage. Cette revue ne prétend pas à l'exhaustivité, mais veille à couvrir la variété la plus large possible de cas de dessertes à grande vitesse tant dans leur forme (desserte sur ligne à grande vitesse ou sur ligne classique, desserte de gare centre ou de gare nouvelle, etc.) que dans l'espace (pays, mais aussi régions et villes) et le temps (de la première desserte à grande vitesse au Japon jusqu'à aujourd'hui, (tableau 1)) ${ }^{1}$.

Tableau 1. Tableau La répartition de la littérature étudiée

\begin{tabular}{|c|c|}
\hline Littérature académique & Littérature non académique \\
\hline $5,6,7,9,10,11,12,13,14,15,16,17,18$, & $1,2,3,4,8,21,23,24,26,36,37,38,40$, \\
$19,20,22,25,27,28,29,30,31,32,33,34$, & $45,52,56,57,65,67,68,72,74,75,76,77$, \\
$35,39,41,42,43,44,46,47,48,49,50,51$, & $78,79,81,82,89$ \\
$53,54,55,58,59,60,61,62,63,64,66,69$, & \\
$70,71,73,80,83,84,85,87,88,90,91,92$ & \\
\hline
\end{tabular}

Source : Réalisé par les auteurs.

Nous désignons par littérature non académique l'ensemble des productions établies à la demande d'un commanditaire, le plus souvent à la demande d'acteurs territoriaux, institutionnels, politiques, économiques qui vont accueillir (études ex-ante) ou qui ont accueilli (ex-post) une desserte ferroviaire à grande vitesse sur leur territoire, et/ou des acteurs qui financent les infrastructures liées à cette desserte (les collectivités locales, les opérateurs de transport, etc.). La littérature académique est quant-à-elle une littérature

\footnotetext{
${ }^{1}$ En dehors de l'anglais et dans une moindre mesure de l'espagnol, la littérature en langue étrangère est moins représentée dans notre revue. Cela est particulièrement vrai pour la littérature non académique qui est rédigée en règle générale dans la langue du pays. De même, compte tenu du caractère plus récent de l'informatisation des revues et rapports, la littérature non française la plus ancienne est sous-représentée.
} 
publiée dans des revues académiques, des ouvrages et/ou présentées dans des colloques universitaires.

L'examen de ces deux types de littérature révèle qu'elles identifient toutes deux de nombreux effets associés à ces dessertes à grande vitesse, selon des catégories relativement similaires. II est ainsi possible de distinguer des effets sur les transports (1.1), sur la structure spatiale des territoires à différentes échelles (1.2), sur les entreprises et l'activité économique (1.3) et enfin des effets généraux et sur les ménages (1.4). Mais la littérature non académique étudie relativement peu les effets sur l'offre de transport alors que la littérature académique en fait une condition de l'émergence des effets sur la demande. Par ailleurs, dans l'identification des effets, la littérature non académique est beaucoup moins précise et détaillée que la littérature académique, envisageant uniquement quelques grandes catégories, réduisant ainsi la complexité de la question des effets que traduit la multiplication des catégories et des nuances.

\subsection{Les effets sur la demande de transport : une prise en compte inégale de l'intermédiation de l'amélioration de l'offre}

Les deux littératures mettent tout d'abord en évidence les effets d'une desserte à grande vitesse sur l'évolution de l'offre de transport, en termes de coûts ou de destinations desservies (cf. tableau 2) ainsi que sur l'augmentation de la demande (cf. tableau 3). Une infrastructure nouvelle à grande vitesse permet d'améliorer le service de transport, en coût ou en qualité, et, ce faisant, génère ou capte de nouveaux trafics. Ainsi la littérature s'intéresse aux effets de la desserte à grande vitesse sur le coût généralisé du déplacement qui dépend notamment de la réduction des temps de parcours. Cette réduction induit des modifications d'usage des autres modes de transport. La desserte à grande vitesse permet également d'améliorer l'accessibilité et l'offre de destinations.

Tableau 2. La littérature relative aux effets sur l'offre de transport

\begin{tabular}{|l|c|c|}
\hline Type de littérature & Académique & Non académique \\
\hline Effets sur le coût généralisé de transport & $22,32,33,70,85$ & 56 \\
\hline $\begin{array}{l}\text { Réduction des temps de parcours entre grandes } \\
\text { villes }\end{array}$ & $\begin{array}{c}7,8,9,10,11,12,13, \\
14,32,33,47,71,87\end{array}$ & $8,23,68,74$ \\
\hline Réduction du nombre des accidents & 70,87 & \\
\hline Accroissement de la fréquence & 32,71 & \\
\hline Accroissement de la capacité & 22,85 & \\
\hline Effets sur les autres modes & 47,85 & \\
\hline Réorganisation des transports ferroviaires & 22 & \\
\hline $\begin{array}{l}\text { Possibilité de développer les capacités } \\
\text { aériennes }\end{array}$ & $22,71,87$ & \\
\hline Réduction de la congestion des aéroports & $20,31,32,33,47,58,65,76$ \\
\hline Effets sur l'offre de destinations & 85,87 & \\
\hline $\begin{array}{l}\text { Amélioration de l'accessibilité des villes à } \\
\text { d'autres espaces }\end{array}$ & $33,47,71$ & 21 \\
\hline $\begin{array}{l}\text { Augmentation des possibilités d'AR dans la } \\
\text { journée }\end{array}$ & 85 & \\
\hline Accroissement de l'accessibilité aux petites villes & 58 & \\
\hline $\begin{array}{l}\text { Amélioration de l'accessibilité aux régions } \\
\text { périphériques }\end{array}$ & & \\
\hline \multicolumn{1}{|c|}{ Source : Réalisé par les auteurs. } & \\
\hline
\end{tabular}

Parmi les effets sur la demande, on distingue, au côté de l'induction de trafic, ou des modifications de la répartition des parts modales, des effets sur des modifications plus qualitatives des trafics (déplacements professionnels, touristiques, etc.). Dans les deux types de littératures prédominent largement à la fois, les effets liés à l'augmentation de la demande ferroviaire et la captation de trafic aérien. Une forte augmentation des trafics ferroviaires est 
ainsi attendue de reports modaux en provenance de l'aérien et pour les distances-temps les plus faibles d'une augmentation des navetteurs.

Tableau 3. La littérature relative aux effets sur la demande de transport

\begin{tabular}{|l|c|c|}
\hline Type de littérature & Académique & Non académique \\
\hline Induction de trafic & & \\
\hline Augmentation de la demande ferroviaire & $6,42,53,54,80,87$ & $65,68,74$ \\
\hline \begin{tabular}{l} 
Induction de trafic lié au travail métropolitain \\
\hline Trafic induit pour l'aérien
\end{tabular} & 22 & 56 \\
\hline $\begin{array}{l}\text { Modification de la répartition des parts } \\
\text { modales }\end{array}$ & & \\
\hline $\begin{array}{l}\text { Diminution de la demande en ferroviaire } \\
\text { conventionnel }\end{array}$ & 32,47 & \\
\hline $\begin{array}{l}\text { Modification de la répartition des parts } \\
\text { modales }\end{array}$ & $20,22,59$ & \\
\hline $\begin{array}{l}\text { Captation de trafic aérien } \\
\text { Modification des trafics }\end{array}$ & $44,47,54,71,87$ & $56,67,68,75,78,79$ \\
\hline $\begin{array}{l}\text { Augmentation des déplacements } \\
\text { professionnels }\end{array}$ & $20,47,71$ & 4 \\
\hline $\begin{array}{l}\text { Augmentation decements } \\
\text { touristiques }\end{array}$ & 20,47 & \\
\hline $\begin{array}{l}\text { Augmentation de l'usage du ferroviaire en } \\
\text { pré-acheminement aérien }\end{array}$ & 20 & 74 \\
\hline $\begin{array}{l}\text { Augmentation des navetteurs pour les } \\
\text { distances-temps les plus faibles }\end{array}$ & & 68,72 \\
\hline $\begin{array}{l}\text { Augmentation de la mobilité de certaines } \\
\text { catégories d'usagers et de certaines } \\
\text { professions }\end{array}$ & 47 & \\
\hline
\end{tabular}

Source : Réalisé par les auteurs.

Toutefois, si la littérature académique insiste autant sur l'offre que sur la demande, ce n'est pas le cas de la littérature non académique étudiée qui met davantage l'accent sur les effets de demande. Les effets sur les trafics interviendraient alors indépendamment des évolutions de l'offre de transport, ouvrant la voie à une interprétation déterministe des effets des dessertes à grande vitesse sur les flux. En éludant cette intermédiation de l'amélioration de l'offre, envisagée comme implicite, la relation entre une desserte à grande vitesse et les évolutions de trafics apparaît alors comme causale. De plus, la littérature académique semble plus précise, alors que la littérature non académique se limite pour le volet sur l'offre de transport aux effets sur le coût généralisé de transport. Dès lors, les effets sur la demande semblent relever uniquement d'une intermédiation par les coûts : une réduction de ce coût généralisé génèrerait automatiquement un ajustement des schémas de mobilité de tous les acteurs et donc une augmentation ou un report de trafic.

Cependant, force est de constater que le bilan de 25 ans de construction de ligne à grande vitesse en France relativise ces anticipations. Si les gains de temps s'accompagnent d'une augmentation des trafics et de reports modaux, les trafics sont souvent inférieurs de 10 à 20 $\%$ par rapport aux estimations. Les reports modaux concernent par ailleurs des catégories de voyageurs aux revenus élevés qui ont renoncé à utiliser l'avion au profit de la grande vitesse ferroviaire. C'est donc la clientèle des CSP supérieures et intermédiaires qui est la grande bénéficiaire de la grande vitesse. Les professions supérieures des activités de conseils, d'études et d'assistance connaissent ainsi la plus forte croissance de leur mobilité.

L'amélioration de l'accessibilité envisagée par les deux littératures doit elle-même être nuancée. D'une part, les agglomérations sur le réseau conventionnel peuvent être confrontées à une dégradation de leur accessibilité en termes de liaisons directes et de fréquences. D'autre part, les agglomérations desservies ne bénéficient pas toutes de la 
même desserte. Enfin, la littérature non académique n'identifie pas certains effets négatifs que la littérature académique souligne pourtant, et notamment la diminution de la demande en ferroviaire conventionnel.

\subsection{Les effets sur la structuration spatiale : des effets qui font débat}

Globalement, les deux types de littérature semblent considérer de façon plus nuancée les effets sur la structure spatiale, en mentionnant les risques de renforcement des grands pôles urbains au détriment des pôles plus fragiles (tableau 4).

Tableau 4. La littérature relative aux effets sur la structuration spatiale à différentes échelles (Europe, pays, régions, villes, quartiers)

\begin{tabular}{|c|c|c|}
\hline Type de littérature & Académique & Non académique \\
\hline \multicolumn{3}{|l|}{$\begin{array}{l}\text { Renforcement économique des villes et des } \\
\text { grands centres urbains }\end{array}$} \\
\hline Renforcement des grands pôles urbains & $16,35,47,66,69,83$ & 72 \\
\hline Accélération de la métropolisation & $10,35,47,83$ & 72 \\
\hline Développement urbain & 60 & 26,76 \\
\hline $\begin{array}{llll}\begin{array}{l}\text { Intégration } \\
\text { dynamiques }\end{array} & \text { des } & \text { territoires } & \text { périurbains } \\
\end{array}$ & & 26 \\
\hline $\begin{array}{l}\text { Dispersion de la population au détriment des } \\
\text { grands centres urbains }\end{array}$ & 69,71 & \\
\hline $\begin{array}{l}\text { Villes moyennes pénalisées par une faible } \\
\text { desserte }\end{array}$ & & 26,45 \\
\hline \multicolumn{3}{|l|}{$\begin{array}{l}\text { Concentration des effets et des activités } \\
\text { économiques }\end{array}$} \\
\hline Risque d'effets d'agglomération & $\begin{array}{c}18,31,33,34,35 \\
69,71,87\end{array}$ & $26,37,65$ \\
\hline Valorisation des bouts de ligne & 83 & 150 \\
\hline Modification de la localisation des activités & 39 & \\
\hline Concentration des effets et des activités & 50 & \\
\hline \multicolumn{3}{|l|}{ Modification des hiérarchies spatiales } \\
\hline $\begin{array}{l}\text { Pas de modifications de l'armature urbaine } \\
\text { régionale }\end{array}$ & 16 & \\
\hline $\begin{array}{l}\text { Hausse de la concurrence et des inégalités } \\
\text { entre territoires }\end{array}$ & & 26,37 \\
\hline Réduction des inégalités centre/périphérie & $30,33,34,58,91$ & \\
\hline $\begin{array}{l}\text { Renforcement du rôle régional et national des } \\
\text { villes desservies }\end{array}$ & $39,83,85$ & 65 \\
\hline $\begin{array}{l}\text { Renforcement du fonctionnement en réseau } \\
\text { hiérarchisé des espaces métropolitains }\end{array}$ & 42 & \\
\hline Transformation du système des villes & $39,58,85$ & 3 \\
\hline Déformation de l'espace & 32,46 & \\
\hline $\begin{array}{l}\text { Redistribution de la croissance au sein de } \\
\text { l'espace régional }\end{array}$ & 35,39 & \\
\hline \multicolumn{3}{|l|}{ Modification des centres villes } \\
\hline Changement de la structure du centre ville & 47,85 & 3 \\
\hline $\begin{array}{l}\text { Modification de la composition sociale des } \\
\text { centres-villes }\end{array}$ & & $57,72,89$ \\
\hline \multicolumn{3}{|l|}{$\begin{array}{l}\text { Renforcement des gares et de leurs } \\
\text { quartiers }\end{array}$} \\
\hline Développement des gares et de leur quartier & $47,51,60,63$ & $3,45,76$ \\
\hline Rénovation des gares et des quartiers d'affaire & & 11,76 \\
\hline
\end{tabular}
Source : Réalisé par les auteurs. 
La littérature académique insiste pourtant davantage et de façon beaucoup plus précise sur les possibilités de concentration des effets et de modification des hiérarchies spatiales.

La littérature non académique se focalise plutôt sur des problématiques à l'échelle de la ville qu'à l'échelle du territoire, concernant le développement urbain, les modifications des centres-villes et le renforcement des quartiers de gare. Cette focalisation, qui se justifie du point de vue des financeurs des études, fait courir le risque de restreindre les actions d'accompagnement à un échelon local, voire micro local, alors qu'une prise en compte des enjeux et des contraintes à d'autres échelles spatiales s'avèrerait nécessaire.

\subsection{Les effets économiques}

Les effets sur les activités économiques sont les plus représentés dans les deux types de littérature. Ils concernent respectivement la localisation d'entreprises nouvelles ou le développement d'activités économiques existantes (2.3.1) ainsi que le foncier et l'immobilier (2.3.2).

\subsubsection{La desserte ferroviaire à grande vitesse et le développement des activités économiques}

Schématiquement, est attendue la localisation de nouvelles entreprises, et ce en Europe comme aux Etats-Unis, notamment dans les secteurs des technologies de pointe ou tertiaires. Un renforcement de la compétitivité des entreprises et activités existantes est également anticipé, pour l'essentiel dans le domaine du tourisme et plus précisément du tourisme urbain et du tourisme d'affaires - domaine largement investi par les deux types de littérature (tableau 5).

Mais là encore, les effets relevés par la littérature académique semblent plus précis et nuancés que ceux mentionnés par la littérature non académique.

Par ailleurs, la littérature non académique semble mettre l'accent sur le développement exogène lié aux dessertes nouvelles à grande vitesse, alors que la littérature académique considère de façon beaucoup plus équilibrée les éventuelles possibilités de développement endogène relevant d'activités existantes. La focalisation sur le développement exogène de la littérature non académique fait alors courir le risque que les acteurs des territoires se concentrent exclusivement sur des stratégies d'attraction d'entreprises nouvelles au moyen d'une offre de zones ou de parcs d'activités. Ce type de stratégie, qui exacerbe la concurrence entre les territoires, présente deux limites. Premièrement elle omet la nécessité de travailler sur l'ensemble des caractéristiques spécifiques demandées par les entreprises qui cherchent une implantation. Deuxièmement, elle conduit à négliger les entreprises déjà implantées qui constituent pourtant les ressources productives des territoires. 
Tableau 5. Littérature relative à la localisation d'entreprises nouvelles ou au développement d'activités économiques existantes

\begin{tabular}{|c|c|c|}
\hline Type de littérature & Académique & Non académique \\
\hline $\begin{array}{l}\text { Effets sur les entreprises et l'activité } \\
\text { économique }\end{array}$ & 58,88 & \\
\hline \multicolumn{3}{|l|}{ La localisation d'entreprises nouvelles } \\
\hline Accroissement de l'attractivité des territoires & $44,47,88$ & 3,37 \\
\hline $\begin{array}{llll}\text { Accroissement de l'attractivité } & \text { des villes } \\
\text { intermédiaires les plus importantes } & & \\
\end{array}$ & 85 & 72 \\
\hline $\begin{array}{llll}\begin{array}{l}\text { Attractivité pour les entreprises } \\
\text { bouleversée }\end{array} & \text { peu } \\
\end{array}$ & 12 & 68 \\
\hline Localisation de nouvelles entreprises & $39,44,71,85$ & $3,37,40,81$ \\
\hline Localisation d'activités métropolitaines & 85 & \\
\hline $\begin{array}{l}\begin{array}{l}\text { Localisation d'activités de } \\
\text { entreprises }\end{array} \\
\end{array}$ & 85 & \\
\hline Localisation de labos de recherche & 92 & \\
\hline Développement de zones d'activités & & 3,65 \\
\hline $\begin{array}{l}\text { Délocalisation de certaines activités (bureaux) } \\
\text { vers les villes desservies les plus importantes }\end{array}$ & $6,29,69,71,73$ & 65 \\
\hline Echecs de certains parcs d'activité & $27,71,84$ & 72,89 \\
\hline $\begin{array}{l}\text { Développement de projets urbains et } \\
\text { investissements complémentaires }\end{array}$ & 39 & \\
\hline \multicolumn{3}{|l|}{$\begin{array}{l}\text { Amélioration de la compétitivité des } \\
\text { activités existantes }\end{array}$} \\
\hline $\begin{array}{l}\text { Amélioration de la compétitivité des industries } \\
\text { de services }\end{array}$ & 17 & 65 \\
\hline Gains de revenus pour l'industrie du rail & & 65 \\
\hline Extension des aires de marché & & 65 \\
\hline Augmentation des exportations & 30 & \\
\hline Meilleur taux d'occupation des bureaux & 39 & \\
\hline $\begin{array}{l}\text { Accroissement de la productivité et de la } \\
\text { compétitivité }\end{array}$ & 88 & 65 \\
\hline $\begin{array}{l}\text { Développement du tourisme urbain et du } \\
\text { tourisme d'affaires et élargissement du marché } \\
\text { touristique }\end{array}$ & $\begin{array}{c}13,31,39,47,50 \\
71\end{array}$ & $\begin{array}{c}3,4,24,36,45,72 \\
76,82\end{array}$ \\
\hline Développement de réunions d'affaire & 47,85 & \\
\hline Hausse de la fréquentation touristique & 47,71 & 24,82 \\
\hline Saisonnalité qui pourrait être nuancée & & 72 \\
\hline $\begin{array}{l}\text { Diminution du nombre de nuitées et de la durée } \\
\text { des séjours }\end{array}$ & $8,13,47,71$ & 4,36 \\
\hline $\begin{array}{l}\text { Hausse des taux de remplissage des } \\
\text { hébergements }\end{array}$ & & 36 \\
\hline $\begin{array}{l}\text { Elargissement du marché touristique des } \\
\text { grandes agglomérations }\end{array}$ & & 45 \\
\hline $\begin{array}{l}\text { Baisse de la durée des séjours à long terme } \\
\text { (hausse à court terme) }\end{array}$ & 17 & 36 \\
\hline $\begin{array}{l}\text { Renforcement de la concurrence entre les } \\
\text { destinations touristiques }\end{array}$ & 50 & \\
\hline
\end{tabular}
Source : Réalisé par les auteurs.

\subsubsection{La desserte LGV et les effets sur le foncier et l'immobilier}

Une desserte à grande vitesse est également susceptible d'engendrer un certain nombre d'effets sur l'immobilier (tableau 6). Un des mythes les plus pérennes dans ce domaine, 
malgré l'insuffisance des références bibliographiques sur la question, concerne l'augmentation des prix de l'immobilier résidentiel dans les villes desservies. Cette augmentation résulterait d'une attractivité accrue des agglomérations desservies par la grande vitesse pour les ménages. Ces anticipations laissent également envisager le développement économique des espaces desservis puisque les prix immobiliers plus élevés attendus devraient encourager les acteurs économiques à proposer de nouveaux projets immobiliers créateurs de nouveaux emplois.

Tableau 6. Littérature relative aux effets sur le foncier et l'immobilier

\begin{tabular}{|l|c|c|}
\hline Type de littérature & Académique & Non académique \\
\hline Effets sur le foncier et l'immobilier & & $40,74,76$ \\
\hline $\begin{array}{l}\text { Augmentation des prix de l'immobilier de } \\
\text { bureaux }\end{array}$ & 39 & $40,74,76$ \\
\hline Hausse des prix de l'immobilier résidentiel & $25,31,58,71$ & \\
\hline Pas d'effets sur les prix immobiliers & 5 & 57 \\
\hline $\begin{array}{l}\text { Effets contrastés sur les prix immobiliers en } \\
\text { fonction des quartiers }\end{array}$ & 12 & 76 \\
\hline Gentrification des lieux desservis & 12,64 & \\
\hline $\begin{array}{l}\text { Augmentation contrastée des prix } \\
\text { immobiliers résidentiels, des locaux } \\
\text { commerciaux et de bureau }\end{array}$ & & 76 \\
\hline $\begin{array}{l}\text { Diminution contrastée des taux de vacance } \\
\text { des biens commerciaux }\end{array}$ & 12,29 & 21,76 \\
\hline Développement de l'immobilier résidentiel & $11,39,71$ & 21,76 \\
\hline Développement de l'immobilier de bureaux & 35 & 76 \\
\hline Développement de l'immobilier commercial & & \\
\hline
\end{tabular}

Source : Réalisé par les auteurs.

Mais alors que la littérature non académique envisage principalement une possible augmentation des prix de l'immobilier, la littérature académique ne fournit pas de réponse tranchée ; elle relève parfois l'absence d'effets ou des effets contrastés selon les quartiers.

Si généralement, l'augmentation des prix immobiliers est présentée comme un effet positif de la mise en œuvre d'une ligne à grande vitesse, cette progression peut également avoir des conséquences sur la localisation des ménages. En produisant une gentrification des agglomérations et des quartiers les mieux desservis, la littérature non académique indique qu'elle conduirait à un rejet des populations les plus fragiles vers la périphérie loin des gares et des quartiers bien reliés à la nouvelle infrastructure.

Par ailleurs, ces évolutions doivent aussi être analysées au regard des stratégies de rénovation des gares souvent mises en œuvre lors de la mise en place de la desserte à grande vitesse. Les gares deviennent alors de véritables hubs de transport. De plus, ces actions s'intègrent souvent à un projet plus vaste de rénovation urbaine du quartier de la gare ayant comme corollaire le développement de projets immobiliers résidentiels, commerciaux et de bureaux. De véritables quartiers d'affaires viennent alors remplacer les vieux quartiers d'arrière gare.

\subsection{Les effets généraux et les effets sur les ménages}

Une desserte ferroviaire à grande vitesse est fréquemment supposée avoir un impact positif sur l'image de la ville desservie, en termes de modernité et de notoriété. Ainsi en France, le TGV fait beaucoup parler des villes au moment de son arrivée et entraine un effet " club » des villes qu'il dessert.

Cet effet d'image est largement reconnu dans les deux types de littératures (tableau 7). En revanche, les autres effets généraux semblent relever davantage de cas précis. 
Tableau 7.Les effets généraux

\begin{tabular}{|l|c|c|}
\hline Type de littérature & Académique & Non académique \\
\hline Amélioration du bien être social & 70 & \\
\hline Modification de la cohésion régionale & 9 & \\
\hline Développement de la cohésion régionale & 88 & $3,24,45,72$ \\
\hline Changement de l'image de la ville & $16,22,25,39,69$, & 40 \\
\hline Amélioration de la qualité de la vie & $81,87,90$ & \\
\hline Effets psychologiques & 92 & \\
\hline $\begin{array}{l}\text { Accompagne ou amplifie les mutations } \\
\text { économiques contemporaines }\end{array}$ & 41 & \\
\hline \multicolumn{2}{|c|}{ Source : Réalisé par les auteurs. }
\end{tabular}

Au-delà des effets généraux, les deux catégories de littérature identifient un certain nombre d'effets tenant à l'attraction de population, la croissance de l'emploi et l'évolution du marché du travail (tableau 8).

Tableau 8.Littérature concernant les effets sur les ménages et l'emploi

\begin{tabular}{|c|c|c|}
\hline Type de littérature & Académique & Non académique \\
\hline Attraction de population & $32,35,69,71,85$ & 3 \\
\hline Hausse du revenu moyen des ménages urbains & 71 & 89 \\
\hline $\begin{array}{l}\text { Faible impact sur le GDP (de moins de } 1 \% \text { à } \\
3 \% \text { ) }\end{array}$ & 64 & \\
\hline $\begin{array}{l}\text { Faible bouleversement de l'attractivité des } \\
\text { ménages }\end{array}$ & & 68 \\
\hline \multicolumn{3}{|l|}{ Effets sur l'emploi } \\
\hline Création d'emplois indirects & & 81 \\
\hline Croissance de l'emploi dans les villes desservies & $13,32,35,69,71$ & 76,89 \\
\hline Croissance de l'emploi lié au chantier & 31 & \\
\hline Perte d'emplois pour les modes concurrents & 18,31 & \\
\hline $\begin{array}{l}\text { Croissance de l'emploi dans les régions } \\
\text { périphériques }\end{array}$ & 30 & \\
\hline Elargissement du marché du travail & 20,39 & 65 \\
\hline \begin{tabular}{|l|} 
Intégration spatiale des marchés du travail \\
\end{tabular} & 35 & \\
\hline $\begin{array}{l}\text { Mobilisation accrue et plus efficace des } \\
\text { travailleurs hautement qualifiés }\end{array}$ & 35 & 89 \\
\hline $\begin{array}{l}\text { Baisse de la demande pour les travailleurs } \\
\text { moyennement qualifiés }\end{array}$ & 35 & 89 \\
\hline $\begin{array}{l}\text { Modification de la sociologie du marché de } \\
\text { l'emploi }\end{array}$ & & 89 \\
\hline $\begin{array}{l}\text { Diminution des emplois industriels ou de faible } \\
\text { qualification au profit d'emplois de cadres ou à } \\
\text { haute qualification }\end{array}$ & & 89 \\
\hline $\begin{array}{l}\text { Accès amélioré au marché de l'emploi pour les } \\
\text { zones rurales }\end{array}$ & 35 & 89 \\
\hline Hausse des taxes locales pour financer la ligne & & 57 \\
\hline
\end{tabular}

Cette thématique est appréhendée de façon plus diversifiée pour les commanditaires de la littérature non académique. L'écart entre les deux littératures se creuse alors, la littérature non académique développant une vision beaucoup plus positive incluant des améliorations de revenu, la création d'emplois indirects, et une modification de la sociologie du marché de l'emploi au profit des travailleurs les plus qualifiés. Elle élude par ailleurs les possibles pertes d'emplois pour les modes de transport concurrents. 
Ainsi l'examen de ces deux formes de littérature montre qu'elles continuent toutes deux d'identifier de nombreux effets liés aux dessertes à grande vitesse, en se concentrant sur des centres d'intérêt communs (le développement économique, l'emploi, l'aménagement de l'espace, etc.). La problématique de l'effet n'est par conséquent pas abandonnée par la littérature académique, ce qui fournit un autre élément permettant de justifier sa pérennité dans la littérature non académique.

Toutefois, la littérature académique nuance davantage le propos, en révélant des effets contrastés ou inexistants dans certains domaines. Elle développe, d'une part, une analyse plus approfondie, en décomposant les grandes catégories d'effets et en questionnant ce faisant les mécanismes d'apparition de l'effet. Elle se situe, d'autre part, à une échelle spatiale plus importante, alors que la littérature non académique se focalise sur l'urbain.

Enfin, la littérature académique relève le rôle essentiel de l'amélioration de l'offre sur la demande de transport, rôle qui ne se limite pas aux seuls effets de réduction des coûts de transport, mais qui tient également à la qualité de la desserte et à l'amélioration de l'accessibilité. L'effet, loin d'être automatique, devient alors conditionné.

La littérature non académique reconnaît elle aussi l'importance de certaines de ces conditions, et propose de mettre en place pour les satisfaire des stratégies d'accompagnement.

C'est donc à l'analyse comparée des conditions recensées par chaque type de littérature qu'il convient maintenant de s'intéresser.

\section{LES ENSEIGNEMENTS D'UNE CONFRONTATION ENTRE LA LITTERATURE ACADEMIQUE ET NON ACADEMIQUE RELATIVE AUX CONDITIONS D'APPARITION DES EFFETS}

La littérature académique dispose désormais d'un certain recul lui permettant de nuancer les effets des dessertes à grande vitesse. Si elle ne remet pas toujours en cause ces effets, elle conditionne leur apparition à différents éléments, qu'ils soient conjoncturels ou structurels, endogènes ou exogènes. Ainsi pour que des effets positifs associés à l'arrivée d'une desserte à grande vitesse aient lieu, un certain nombre de conditions sont évoquées. Certaines de ces conditions sont reprises par la littérature non académique, qui a bien intégré notamment la nécessité de mettre en place des stratégies d'accompagnement centrales dans l'émergence de certains effets. Nous montrerons néanmoins que la prise en compte des conditions demeure insuffisante, occultant, la plupart du temps, la demande, la conjoncture ou les caractéristiques liées à la ligne ou au service à grande vitesse (3.1). Les conditions relatives à la mise en œuvre de stratégies ne sont également que partiellement reprises. La littérature non académique se focalise sur les stratégies d'accompagnement reposant sur une meilleure desserte ou une offre de transport complémentaire, au détriment des stratégies permettant de coordonner les différents acteurs (3.2). Enfin, le caractère contextualisé de la littérature académique, au sens où de nombreuses conditions tiennent aux caractéristiques intrinsèques du territoire, nous semble insuffisamment pris en compte (3.3).

\subsection{Une prise en compte insuffisante des conditions par la littérature non académique}

La littérature académique identifie de nombreuses conditions nécessaires à l'apparition des effets. Mais celles tenant au contexte macro économique, aux caractéristiques de la ligne ou du service ferroviaire à grande vitesse, ou encore à l'existence d'une demande effective sont souvent occultées par la littérature non académique (tableau 9).

Or, la fréquentation des trains à grande vitesse et les effets sont dépendants de la conjoncture économique et de la croissance économique qui influe sur la demande de transport de personnes, et ce quels que soient les motifs du déplacement. La conjoncture économique influence en outre la stratégie des acteurs publics et privés par les capacités 
d'investissement qu'elle permet ou non. Le niveau de la concurrence intermodale et l'offre tarifaire du transport aérien vont également conditionner les parts de marchés de la grande vitesse ferroviaire par rapport au transport aérien sur les distances moyennes et longues.

Les caractéristiques liées à la ligne ou au service ferroviaire à grande vitesse vont également influer sur la fréquentation et les effets. Parmi ces caractéristiques, les temps de parcours, les fréquences, la vitesse d'exploitation et plus généralement la qualité de l'offre, vont déterminer en partie la demande de transport. La distance entre les villes desservies, et le type de réseau utilisé seront également déterminants dans l'existence ou non d'effets.

Tableau 9. Les conditions liées au contexte macro-économique, au type de ligne ou au service ferroviaire à grande vitesse

\begin{tabular}{|l|c|c|}
\hline Type de littérature & Académique & Non académique \\
\hline Contexte macroéconomique & & 68 \\
\hline Conjoncture économique & $\begin{array}{c}19,20,28,32,35, \\
47,71,85\end{array}$ & \\
\hline $\begin{array}{l}\text { Dérégulation aérienne favorisant la baisse des } \\
\text { coûts aériens }\end{array}$ & 58 & \\
\hline $\begin{array}{l}\text { Caractéristiques liées à la ligne ou au } \\
\text { service ferroviaire }\end{array}$ & & \\
\hline Caractéristiques de la ligne & & 3 \\
\hline Caractéristiques techniques de la gare & 32,85 & 65 \\
\hline Type de HSR & $20,28,32,87$ & 76 \\
\hline Nombre d'arrêts & 53,64 & \\
\hline Création de nouvelles voies dédiées & 32,39 & \\
\hline Distance entre les villes desservies & $32,33,85,88$ & \\
\hline Caractéristiques du service & $6,54,85$ & \\
\hline Temps de parcours & 20,59 & 57,77 \\
\hline Vitesse d'exploitation & $20,44,53,58,59,85$ & \\
\hline Amélioration des fréquences & 20 & \\
\hline Prix & & 20 \\
\hline Incitations tarifaires & 20 & \\
\hline Politique tarifaire spécifique pour les touristes & 53 & \\
\hline Système d'achats de billets sans réservation & 20 & \\
\hline Existence de ventes de billets en centre-ville & & \\
\hline Qualité du service de transport & & \\
\hline Politique marketing des autres modes \\
(autocars)
\end{tabular}

Source : Réalisé par les auteurs.

La mise en place d'une desserte ferroviaire à grande vitesse conduit à une modification des parts de marché entre l'aérien et le ferroviaire pour les distances comprises entre 200 et 700 $\mathrm{km}$ ou pour des durées de transport entre une et trois heures (tableau 10). L'utilisation de cette desserte résulte ainsi principalement de report de trafics et dans une moindre mesure de trafics induits par sa mise en place. Aussi des trafics préexistants sont-ils nécessaires pour permettre une fréquentation.

La politique «marketing » menée par l'exploitant du réseau à grande vitesse est également une condition cruciale pour en assurer le succès. En effet, la desserte s'accompagne d'une augmentation du coût de déplacement (augmentation du prix du billet) qui peut être compensée par des temps de parcours plus faibles grâce à l'augmentation des vitesses mais également par l'augmentation des fréquences de la desserte. II s'agit donc pour l'exploitant d'inciter à utiliser la desserte, en facilitant l'achat de billets, voire en proposant une politique tarifaire différenciée. C'est le cas pour les déplacements touristiques de familles, afin de compenser le caractère onéreux du ferroviaire par rapport à la voiture particulière. 
Tableau 10. Les conditions liées à l'existence d'une demande effective

\begin{tabular}{|l|c|c|}
\hline Type de littérature & Académique & Non académique \\
\hline Existence d'une demande effective & 54,91 & 65 \\
\hline $\begin{array}{l}\text { Demande de mobilité suffisante dans un rayon } \\
\text { de } 300 \text { à } 600 \mathrm{kms}\end{array}$ & 70,85 & \\
\hline $\begin{array}{l}\text { Existence d'une demande spécifique en } \\
\text { distance (200-700kms) et temps (1h-3h) }\end{array}$ & $20,59,88$ & \\
\hline Demande préalable effective & 44 & \\
\hline Trafic conforme aux prévisions & 18 & 68 \\
\hline Existence du report modal & & \\
\hline \multicolumn{2}{|l}{ Source : Réalisé par les auteurs. }
\end{tabular}

\subsection{Une reconnaissance inégale des stratégies d'accompagnement}

La conditionnalité des effets justifie la mise en place de stratégies d'accompagnement. Leur nécessité est reconnue par la littérature non académique, qui insiste notamment sur les stratégies visant à améliorer la desserte ou à construire une offre de transport complémentaire (3.2.1). Néanmoins, la littérature académique détaille de façon beaucoup plus précise, d'une part, ces stratégies d'accompagnement, et évoque, d'autre part, l'importance du contexte dans lequel ces stratégies s'inscrivent. C'est le cas notamment quand elle souligne la nécessaire coopération de tous les acteurs, ou encore l'existence de cadres de coopération ou d'institutions appropriées (3.2.2).

\subsubsection{Une reconnaissance sensiblement équivalente de la nécessité de stratégies de transport en matière d'accompagnement}

Les stratégies de transport, qui sont relevées par les deux types de littérature, s'articulent autour de deux grandes actions prioritaires : l'amélioration de l'interconnexion entre les réseaux et les réflexions tenant au renforcement de l'accessibilité à la gare ou plus largement à la ville et nécessitant des investissements complémentaires en infrastructures ou en services (tableau 11).

Tableau 11. Les conditions liées à l'amélioration des interconnexions

\begin{tabular}{|l|c|c|}
\hline Type de littérature & Académique & Non académique \\
\hline Investissement (infrastructures et services) & 30,31 & \\
\hline Amélioration de l'interconnexion entre modes & & $2,52,68,72,75$ \\
\hline $\begin{array}{l}\text { Bonne interconnexion aux aéroports et aux gares } \\
\text { ferroviaires classiques }\end{array}$ & $\begin{array}{c}5,20,22,69,76, \\
83,88,71,87\end{array}$ & 82 \\
\hline Interconnexion avec les Transports Collectifs & $54,71,83$ & \\
\hline $\begin{array}{l}\text { Interconnexion Grande vitesse ferroviaire -voies } \\
\text { rapides }\end{array}$ & 35 & $3,57,77$ \\
\hline $\begin{array}{l}\text { Bonne desserte ferroviaire à partir des villes } \\
\text { bénéficiant de la Grande vitesse ferroviaire }\end{array}$ & $30,33,85$ & 3 \\
\hline Qualité de la nouvelle desserte & $30,47,88$ & 65 \\
\hline Qualité de la desserte préexistante & $6,30,71,85,87$ & \\
\hline Concurrence légère avec les autres modes & 80 & \\
\hline $\begin{array}{l}\text { Gestion de la rupture de charge quand la gare } \\
\text { n'est pas au centre ville }\end{array}$ & 92 & \\
\hline
\end{tabular}
Source : Réalisé par les auteurs

L'interconnexion entre les modes permet en effet d'améliorer la demande pour les services à grande vitesse en limitant les contraintes liées à la correspondance dans les gares et les aéroports. Dans ce domaine, les deux catégories de littératures semblent dresser des analyses sensiblement équivalentes.

Plus largement, l'amélioration de la connexion entre villes ou de l'accessibilité à la ville desservie et à la gare s'avère également déterminante (cf. tableau 12). 
Tableau 12. Les conditions liées à l'amélioration de l'accessibilité

\begin{tabular}{|l|c|c|}
\hline Type de littérature & Académique & Non académique \\
\hline Amélioration de la connexion entre les villes & & \\
\hline Bonne connexion des grandes villes entre elles & $33,54,83,87$ & \\
\hline $\begin{array}{l}\text { Amélioration de l'accessibilité des petites et } \\
\text { moyennes villes }\end{array}$ & 34,88 & \\
\hline Amélioration de l'accessibilité interrégionale & 20 & $40,76,81$ \\
\hline $\begin{array}{l}\text { Intégration des villes intermédiaires dans les } \\
\text { corridors }\end{array}$ & 85 & 40 \\
\hline Interconnexion des marchés & & \\
\hline $\begin{array}{l}\text { Amélioration de l'accessibilité à la ville et à la } \\
\text { gare }\end{array}$ & 83 & \\
\hline Accessibilité à la ville & 18 & 76 \\
\hline Traiter les points de congestion autour des villes & $19,20,59,60,69$ & \\
\hline Amélioration de l'accessibilité à la gare & $35,47,54,64,71$, & $3,72,81,89$ \\
\hline $\begin{array}{l}\text { Localisation de la gare } \\
\text { Degré d'insertion de la gare dans le tissu urbain }\end{array}$ & $83,85,88$ & \\
\hline
\end{tabular}

Source : Réalisé par les auteurs

Elle dépend de la nature de la desserte organisée, de la qualité de l'interconnexion des villes desservies, ou de l'organisation de l'accessibilité des petites et moyennes villes.

La localisation de la gare tout comme son accessibilité - associées au développement des transports en commun, des accès piétons et en vélos, ou des parkings - sont aussi des conditions évoquées par les deux littératures. Au sein des villes, la bonne connexion avec les transports collectifs est ainsi déterminante pour limiter les difficultés associées à la rupture de charge. Cette accessibilité à la gare s'avère cruciale et ce en tout point des agglomérations desservies : il est en effet important que les temps d'accès à la gare n'annulent pas les gains de temps liés à l'usage de la grande vitesse. Les analyses convergent enfin sur la difficulté des gares excentrées - par rapport aux gares en centre-ville - à être bien reliées à l'ensemble du territoire desservi.

Si ces stratégies liées à l'accompagnement de la desserte sont reconnues dans les deux catégories de littérature, d'autres stratégies le sont de façon différente.

\subsubsection{Une intégration différente du contexte stratégique et des autres stratégies d'accompagnement}

Outre les stratégies de transport, d'autres stratégies d'accompagnement visant par exemple à restructurer la gare et son quartier sont également évoquées par les deux types de littérature (tableau 13). Ainsi, avant la mise en service de la grande vitesse ferroviaire, les gares sont rénovées et parfois transformées en hubs pour les différents flux de transports et/ou en zones commerciales. Au-delà des gares elles-mêmes, ces évolutions concernent leurs quartiers qui sont souvent des quartiers considérés comme peu fréquentables et peu sûrs. Une partie des politiques publiques d'accueil d'une desserte à grande vitesse consiste alors à améliorer leur image. De nombreux projets de réhabilitation et de reconstruction de l'immobilier sont ainsi menés transformant ces quartiers en quartier d'affaires ou en zones commerciales. Ces évolutions participent à améliorer la fréquentation de ces zones et leur accessibilité.

Toutefois la littérature académique met en évidence des conditions liées aux réussites des stratégies d'accompagnement que n'évoque pas la littérature non académique. Par exemple, cette dernière tient peu compte du fait que l'aménagement des quartiers des gares excentrées est souvent un échec. En revanche, en soulignant que les activités de services auxquelles sont destinés ces aménagements recherchent plutôt la centralité et les aménités 
liées à la proximité du centre urbain, la littérature académique met en exergue l'importance de la localisation en centre-ville des gares accueillant la grande vitesse ferroviaire.

Tableau 13.Les conditions liées au contexte stratégique

\begin{tabular}{|l|c|c|}
\hline Type de littérature & Académique & Non académique \\
\hline Stratégies & $18,50,53,64$ & $2,37,45,68,72,89$ \\
\hline Contexte stratégique & & $1,37,68,89$ \\
\hline Concertation des acteurs & $7,13,31,85$ & $3,4,8,21$ \\
\hline Coopération de tous les acteurs publics et privés & & 3 \\
\hline Existence de cadres de coopération & 85 & \\
\hline Poids politique de la région & 85 & \\
\hline Existence d'institutions appropriées & 69 & \\
\hline $\begin{array}{l}\text { Cohérence avec les stratégies des firmes et du } \\
\text { marché de l'emploi }\end{array}$ & 85 & 89 \\
\hline Existence de projets urbains préalables & & \\
\hline Nature des stratégies & & $21,37,40$ \\
\hline $\begin{array}{l}\text { Anticiper/accompagner les mutations de l'emploi } \\
\text { en menant des politiques publiques volontaristes } \\
\text { (enseignement supérieur, formation) }\end{array}$ & & $21,38,89$ \\
\hline $\begin{array}{l}\text { Accompagner en les anticipant les mutations } \\
\text { immobilières associées à l'arrivée d'une LGV }\end{array}$ & & \\
\hline Promouvoir les spécificités des territoires & $13,50,71$ & \\
\hline $\begin{array}{l}\text { Stratégies de développement des tourismes } \\
\text { d'affaires et urbain }\end{array}$ & 13,50 & 21,76 \\
\hline Différenciation des produits touristiques & 71,85 & 3,21 \\
\hline Politique marketing des collectivités locales & 60 & \\
\hline Libération de foncier au centre ville & 20,71 & \\
\hline Restructuration des gares & 85 & \\
\hline Investissement public autour des gares & & \\
\hline Aménagement concerté du quartier de la gare & & \\
\hline Développement de nouveaux projets urbains & & \\
\hline
\end{tabular}

Source : Réalisé par les auteurs.

Par ailleurs, si la nécessité d'une concertation et d'une coopération entre les acteurs locaux sont évoqués par les deux types de littérature, la littérature non académique se limite à l'influence du contexte stratégique local, négligeant ainsi le rôle des contextes stratégiques définis à des échelles spatiales plus importantes. Or par exemple, le poids politique de la région est susceptible d'influer sur la capacité des acteurs locaux à tirer profit d'une desserte à grande vitesse.

\subsection{Les contraintes liées aux caractéristiques du territoire : une contextualisation des effets bien souvent omise par la littérature non académique}

La littérature académique conditionne également son analyse aux caractéristiques intrinsèques des territoires. Celles-ci relèvent de considérations propres aux villes et à leur localisation (3.3.1), ou de la présence de ressources économiques ou liées à la population (3.3.2).

\subsubsection{Des conditions liées au type de ville ou à leur localisation}

Si les conditions relatives à l'influence du type de ville sont nombreuses dans la littérature académique, seules quelques références de la littérature non académique retiennent la taille des villes et leur localisation comme facteurs importants d'apparition des effets (tableau 14). De surcroît, les conditions évoquées sont une fois encore beaucoup moins précises. Ainsi la littérature non académique n'évoque pas le rôle de la distance entre les villes pas plus que celui de la proximité de centres urbains plus importants. 
Tableau 14. Les contraintes liées aux types de ville et à leur localisation

\begin{tabular}{|l|c|c|}
\hline Type de littérature & Académique & Non académique \\
\hline Type de villes et distance entre elles & & \\
\hline Importance du centre urbain & $69,87,88$ & \\
\hline Densité de population des villes & $32,44,80,85,91$ & \\
\hline $\begin{array}{l}\text { Appartenance des villes à la même région } \\
\text { administrative }\end{array}$ & 85 & \\
\hline Taille et localisation des villes & $32,64,85$ & 2,72 \\
\hline Distance comprise entre 200 et $500 \mathrm{~km}$ & 64 & \\
\hline $\begin{array}{l}\text { Distance à une grande ville inférieure ou égale à } \\
\text { 2h }\end{array}$ & 69,88 & \\
\hline Localisation centrale ou périphérique en Europe & 34,8 Source : Réalisé par les auteurs.
\end{tabular}

\subsubsection{Les conditions liées aux ressources du territoire}

Dans ce domaine l'écart entre les deux types de littérature est important (tableau 15).

Tableau 15. Les contraintes liées aux ressources du territoire

\begin{tabular}{|c|c|c|}
\hline Type de littérature & Académique & Non académique \\
\hline \multicolumn{3}{|l|}{ Ressources économiques } \\
\hline Taille des marchés de la ville & 88 & \\
\hline $\begin{array}{l}\text { Degré d'agglomération des activités pour } \\
\text { permettre les économies d'échelle }\end{array}$ & 69,88 & \\
\hline $\begin{array}{llll}\begin{array}{l}\text { Entreprises } \\
\text { international }\end{array} & \text { disposant } & \text { d'un } & \text { marché } \\
\end{array}$ & 47 & \\
\hline $\begin{array}{l}\text { Présence d'activités économiques reposant } \\
\text { sur l'échange d'informations }\end{array}$ & 35 & \\
\hline Présence d'universités & 35 & \\
\hline Existence de services de qualité & 85 & \\
\hline Existence préalable de liaisons économiques & $30,39,47$ & \\
\hline $\begin{array}{l}\text { Existence de centres d'affaires denses et } \\
\text { centraux }\end{array}$ & 80 & \\
\hline $\begin{array}{l}\text { Ressources touristiques initiales suffisantes et } \\
\text { adaptées à l'usage du train }\end{array}$ & $17,47,50$ & \\
\hline Niveaux de productivité suffisants & 30 & \\
\hline Economie locale dynamique & $18,31,32,47,71$ & \\
\hline $\begin{array}{l}\text { Existence d'un potentiel économique et } \\
\text { d'activités économiques sur le territoire }\end{array}$ & 69,71 & $36,68,72$ \\
\hline $\begin{array}{l}\text { Accessibilité à des villes présentant un } \\
\text { volume d'activité économique important }\end{array}$ & 33 & \\
\hline Image de la ville et attractivité & 63,85 & \\
\hline \multicolumn{3}{|l|}{ Caractéristiques du foncier } \\
\hline Coûts de l'immobilier & 12,85 & \\
\hline Disponibilité foncière au centre ville & $11,12,71$ & $3,21,76$ \\
\hline $\begin{array}{l}\text { Concurrence entre immobiliers résidentiels et } \\
\text { immobiliers de bureaux }\end{array}$ & $-3=-5$ & \\
\hline \multicolumn{3}{|l|}{ Ressources liées à la population } \\
\hline Type d'habitants & 29 & \\
\hline Part du travail métropolitain & 42 & \\
\hline Coûts salariaux plus faibles & 85 & \\
\hline Existence d'une main d'œuvre qualifiée & 30 & \\
\hline
\end{tabular}


La littérature académique identifie de multiples conditions spécifiques pour que des effets se produisent. tandis que, la littérature non académique, moins précise sur les caractéristiques spécifiques des territoires où des effets se produisent, retient simplement l'existence d'un potentiel économique sur les territoires accueillant cette desserte. Ainsi, dans le domaine du tourisme, si une desserte ferroviaire à grande vitesse peut être utilisée par les touristes pour visiter des lieux déjà connus et bénéficiant d'une certaine renommée, la littérature académique met en évidence l'importance de ressources spécifiques reconnues.

La diversité des conditions d'apparition des effets évoquées par la littérature académique présente pourtant une valeur heuristique importante. En effet, elle met clairement en évidence l'absence de modèle unique, d'une loi économique permettant de rendre compte des liens entre desserte à grande vitesse et développement économique local. Les conditions évoquées sont ainsi autant de facteurs limitant les possibilités de transfert des conclusions obtenues dans un contexte donné à d'autres espaces ou d'autres territoires.

\section{CONCLUSION}

Cette communication propose une confrontation entre littérature académique et non académique afin d'éclairer les raisons de la pérennité du mythe des effets structurants d'une ligne à grande vitesse sur les transformations socio-économiques dans l'espace. Nous avons montré que la littérature académique ne permet pas d'évacuer ce mythe et, dans une certaine mesure, participe même à son maintien. Elle continue en effet d'identifier des effets, même si ceux-ci, complexes et nuancés, sont présentés comme dépendants de conditions spécifiques aux territoires desservis.

$\mathrm{Si}$ la complexité et les nuances relatives aux effets, ainsi que certaines conditions importantes, sont parfois absentes des travaux de la littérature non académique, la pérennité du mythe de l'effet ne peut toute entière être imputée à une prise en compte imparfaite des conclusions de la littérature académique. En effet, si ce point constitue un premier élément d'explication, au-delà, nous voudrions également insister sur le fait que le paradigme de la conditionnalité introduit par la littérature académique change peu de choses. II laisse en effet à penser qui si les conditions sont réunies, alors les effets se produiront. Le mythe des effets peut dès lors subsister, en autorisant la généralisation de conclusions pourtant élaborées dans des cas particuliers en termes de conjoncture économique, de localisation des villes desservies dans le réseau de villes, de qualité de desserte, de fréquentation, de ressources propres à chaque territoires et de stratégies des acteurs, etc.

Sauf à produire une analyse précise de la contextualisation de ces effets, la littérature académique participe ce faisant indirectement à pérenniser le mythe.

\section{BIBLIOGRAPHIE}

1. Conseil Regional de Champagne-Ardenne, 2007, Réunion conjointe des Comités Locaux d'Animation et de Développement (CLAD) Ardennes et Charleville-Givet, www.crchampagne-ardenne.fr/getFile.aspx?FILEID=2678

2. Agence de deVeloppement et D'URBAnisme de Strasbourg (ADEUS), 2008, TGV et synergies de développement en Alsace. Diagnostic/enjeux/indicateurs, pour "résumé" de l'ADEUS, http://www.adeus.org/Etudes/Definitions_Politiques_Publiques/resume-d-etudetgv-et-synergies-de-developpement-en-alsace-diagnostic-enjeux-indicateurs

3. AGENCES D'URBANISME DU GRAND-Est, 2005, Les impacts territoriaux du TGV Est et du TGV Rhin-Rhône pour les agglomérations du Grand-Est de la France, 0, www.adumontbeliard.fr/.../364.etu_ex_impacts_tgv_est_rhin_rhone_050705.pdf

4. AMIARD, D., (1997), "Le tourisme d'affaire et de Congrès dans l'agglomération mancelle », in Chevalier J., 1997, Le Mans 6 ans après l'arrivée du TGV, groupe de recherche en 
géographie sociale, ESO - Espaces géographiques et Sociétés, Université du Maine, Le Mans.

5. ANDERSSON, DAVID EMANUEL; SHYR, OlivieR F ; FU, JOHNSON, 2010, Does high-speed rail accessibility influence residential property prices ? Hedonic estimates from southern Taiwan, Journal of Transport Geography (vol. 18, $\mathrm{n}^{\circ 1}$ ) pp. 166-174

6. BARRON I, 2009, High Speed lines in the World, UIC High Speed Department, Updated 14 June http://www.vialibre.org/PDF/4555_AV_en_el_mundo.pdf

7. BAZIN, SYLVIE ; BECKERICH, CHRISTOPHE ; DELAPLACE, MARIE ; MASSON, SOPHIE, 2006a, "L'arrivée de la LGV en Champagne-Ardenne et la nécessaire réorganisation des rapports de proximité ", les Cahiers Scientifiques des transports, $n^{\circ} 49$

8. BAZIN, SYLVIE ; BECKERICH, ChRISTOPHE ; DELAPLACE, MARIE, 2006B, « Analyse prospective des impacts de la Ligne Grande Vitesse Est-Européenne dans l'agglomération rémoise et en région Champagne-Ardenne " rapport final remis à la région ChampagneArdenne, Février, 495 pages + annexes

9. BAZIN, SYLVIE ; BECKERICH, CHRISTOPHE ; DELAPLACE, MARIE ; MASSON, SOPHIE, 2006c, "La LGV-Est Européenne en Champagne-Ardenne : quels effets sur la cohésion territoriale champardennaise ", Revue d'Economie Régionale et Urbaine, N² pp. 245-261

10. BAZIN, SYLVIE ; BECKERICH, CHRISTOPHE ; DELAPLACE, MARIE; MASSON, SOPHIE, 2006d, La Ligne Grande vitesse Est-européenne en région Champagne-Ardenne : Un outil au service d'un processus de Métropolisation ? ", Recherche, Transports et Sécurité $n^{\circ} 92$

11. BAZIN, SYLVIE ; BECKERICH, CHRISTOPHE ; DELAPLACE, MARIE, 2009, « Desserte TGV et localisation des entreprises sur les quartiers d'affaires: nouvelle accessibilité ou nouvelle offre immobilière de bureaux ? Le cas de la gare centre de Reims ", Les Cahiers Scientifiques des Transports, $N^{\circ} 56$ pp. 37-61

12. BAZIN, SYLVIE ; BECKERICH, CHRISTOPHE ; DELAPLACE, MARIE, 2010, « Ligne à Grande Vitesse et marchés immobiliers résidentiels à Reims : entre attractivité, aménités et anticipations », Revue d'Economie Régionale et Urbaine, №2, p. 313-336

13. BAZIN, SYLVIE; BECKERICH, CHRISTOPHE; DELAPLACE, MARIE, (accepté pour publication), High speed railway, service innovations and urban and business tourisms development, in SARMENTO M. \& MATIAS A. "Tourism Economics and Management: The State of the Art", Springer Verlag

14. BLANQUART, CORINNE ; DELAPLACE, MARIE, 2009, « Innovations relationnelles, nouvelles offres de service et valorisation des nouvelles infrastructures de transport, Le cas de d'une plateforme multimodale et d'une desserte TGV » Les Cahiers Scientifiques des Transports, N॰56 pp. 63-86

15. BONNAFOUS, ALAin ET PLASSARD, FrançoIs, 1974, Les méthodologies usuelles de l'étude des effets structurants de l'offre de transport, Revue économique, vol. $\mathrm{XXV}, \mathrm{n}^{\circ} 2$, mars, pp. 208-232

16. BONNAFOUS, AlAIN, 1980, Rhône-Alpes, capitale Paris ? Les effets prévisibles du TGV, Revue de géographie de Lyon (vol. 55, n³), pp. 233-240,

17. BONNAFOUS, ALAIN, 1987, The regional impact of the TGV, Transportation (vol. 14, $\mathrm{n}$ \%), pp. $127-137$

18. BUTTON, KENNETH, 1990, The Channel Tunnel: The Economic Implications for the South East of England, The geographical journal (vol. 156, $\mathrm{n}$ ²), pp. 187-199

19. CHANG, JUSTIN S; LEE, JANG-Ho, 2008, Accessibility analysis of Korean high-speed rail : a case study of the Seoul metropolitan area, Transport reviews (vol. 28, n¹), pp. 87-103 
20. CHENG, YUNG-HSIANG, 2009, High-speed rail in Taiwan: New experience and issues for future development, Transport policy (vol. 17, n²) pp. 51-63

21. CHEVALIER, JEAN, (1997), Le Mans 6 ans après l'arrivée du TGV, groupe de recherche en géographie sociale, ESO - Espaces géographiques et Sociétés, Université du Maine, Le Mans.

22. CHI, ARNAUD, 2004, Do high-speed trains really promote interconnected airports? Laboratoire d'économie des Transports

23. Colin Buchanan in ASSOCiation With VolterRa, 2009, Economic impact of high-speed 1. Final report, pour London \& continental railways,

http://www.colinbuchanan.com/uploads/cms/files/147e7dfc-2a53-4267-83d7-72bdde92062e.pdf

24. Communaute URBaine De Strasbourg, 2007/2008, Rubrique Vie internationale: "Eté et TGV : des effets positifs pour le tourisme », CUS Magazine n`35,

25. DE JONG, MIG, 2009, European high-speed train station area : the renaissance of the railway station, pour The Association for European Transport and contributors, www.etcproceedings.org/paper/download/3918

26. DIACT, 2009, Transport et accessibilité des villes moyennes. Rencontre nationale des villes moyennes - Le Puy-en-Velay, le 11 Septembre 2009 http://www.datar.gouv.fr/IMG/Fichiers/KIOSQUE/2009/20090911_Le-Puy_CR_colloque_ national_villes_moyennes.pdf

27. FACCHINETTI-MANNONE, VALÉRIE, 2009, Location of high speed rail stations in french medium-size city and their mobility and territorial implications: central, peripheral and bis (both central and peripheral in the same city), International Conference City Futures 2009, Madrid, 4-6 june

28. FROIDH, OSKAR, 2005, Market effects of regional high-speed trains on the Svealand line, Journal of transport geography (vol. 13, $\mathrm{n}^{\circ}$ ) pp. 352-361

29. GARMENDIA, Maddi ; URENA, José M de ; RIBALAYGUA, Cecilia ; LEAL, Jesus ; CORONADO, José M, 2008, Urban residential development in isolated small cities that are partially integrated in metropolitan areas by high-speed train, European urban and regional studies (vol. 15, n³) pp. 249-264

30. GIBB, R. A ; KNOWLES, R. D ; FARRINGTON, J. H, 1992, The Channel Tunnel Rail Link and Regional Development: An Evaluation of British Rail's Procedures and Policies, The geographical journal (vol. 158, $n$ ³) pp. 273-285

31. GIBB, R. A, 1986, The impact of the Channel Tunnel rail link on South East England, The geographical journal (vol. 152, n³)

32. GIVONI, MOSHE, 2006, DEVELOPMENT and impact of the modern high-speed train: a review, Transport reviews (vol.26, $\mathrm{n}^{\circ 5}$ ) pp. 593-211

33. GUTIERREZ, JAVIER, 2001, Location, economic potential and daily accessibility: an analysis of the accessibility impact of the high-speed line Madrid-Barcelona-French border, Journal of transport geography (vol. 9, n4) pp. 229-242

34. GutierReZ, JAVIER; GONZALEZ RAFAEL; GOMEZ, GaBRIEL, 1996, The European highspeed train network. Predicted effects on accessibility patterns, Journal of transport geography (vol. 4, n4), pp. 227-238

35. HAYNES, KINGSLEY E., 1997, Labor markets and regional transportation improvements: the case of high speed trains. An introduction and review, The annals of regional science (vol.31, n'1) pp. 57-76 
36. INSEE LORRAINE, 2009, La ligne à Grande Vitesse Est-européenne : une évaluation de l'impact sur le tourisme, Economie - INSEE Lorraine $\mathrm{n}^{\circ} 163$, http://www.insee.fr/fr/insee_regions/lor/themes/el/el163/el163.pdf

37. ISIS, 2004, Analyse de l'impact du TGV-Est sur les agglomérations de Metz, Nancy, Epinal et Thionville. Rapport de phase 2, pour ADIELOR ?, http://www.lorraine.equipement.gouv.fr/IMG/pdf/Rapport_Phase_2_cle214854.pdf

38. ISIS, 2004, Analyse de l'impact du TGV-Est sur les agglomérations de Metz, Nancy, Epinal et Thionville. Rapport de phase 3, pour ADIELOR ?, http://www.lorraine.equipement.gouv.fr/IMG/pdf/Rapport_Phase_3_cle2c9d11.pdf

39. KAMEL, Karima ; MATTHEWMAN, Richard, 2008, The non-transport impacts of High-Speed Trains on regional economic development : a review of the literature, Locate in Kent, http://www.locateinkent.com/images/assets/High\%20Speed\%20Train\%20Report\%202008.p df

40. KANTOR, SHAWN, 2008, The economic impact of the California high-speed rail in Sacramento/Central Valley Area,

http://www.cahighspeedrail.ca.gov/images/chsr/20081003135956_HSRCentralValleyReportF INAL-2.pdf

41. KLEIN, OLIVIER, 2001, La genèse du TGV, une innovation contemporaine de l'épuisement du fordisme, Innovations, Cahiers d'économie de l'innovation (vol. 13, n¹) pp. 111-132

42. KLEIN, OLIVIER, 2003, Le travail métropolitain : un outil géographique pour révéler l'usage sélectif de la grande vitesse, L'espace géographique $\left(n^{\circ 2 / 03)}\right.$

43. KLEIN, OLIVIER, 1997, Le TGV-Atlantique et les évolutions de la mobilité : entre crise et

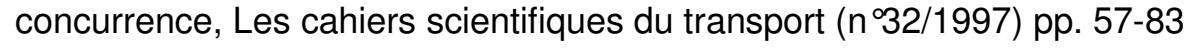

44. LEE, YONG SANG, 2007, A study of the development and issues concerning high speed rail (HSR), Transport Studies Unit, Oxford University Centre for the Environment, http://www.tsu.ox.ac.uk/pubs/1020-lee.pdf

45. Les VILLES EUROPEenNeS DE LA GRANDE VITESSE, 2004, Compte-rendu du débat public : "TGV : 20 ans d'expérience - quels enseignements pour l'Alsace ?", http://pagespersoorange.fr/asso-villestgv/documents/CR_debat-public_CES-Alsace_11-10-04.pdf

46. L'HOSTIS, ALAIN, 2009, The shrivelled USA: representing time-space in the context of metropolitanization and the development of high-speed transport, Journal of transport geography (vol. 17, $\mathrm{n}^{\circ} \mathrm{6}$ ) pp. 433-439

47. MANNONNE, VALERIE, 1995, L'impact régional du TGV sud-est, Thèse pour l'obtention du doctorat de géographie, 2 tomes, Université de Provence Aix-Marseillel

48. MANNONE, VALERIE, 1997, Gares TGV et nouvelles dynamiques urbaines en centre ville : le cas des villes desservies par le TGV Sud-Est, Les cahiers scientifiques du transport (n 31/1997) pp. 71-97

49. MARTIN, JUAN CARLOS; REGGIANI, AuRA, 2007, Recent methodological developments to measure spatial interaction: synthetic accessibility indices applied to high-speed train investments, Transport reviews (vol. 27, n ^5) pp. 551-571

50. MASSON, SOPHIE; PETIOT, ROMAIN, 2009, Can the high speed rail reinforce tourism attractiveness ? The case of the high speed rail between Perpignan (France) and Barcelona (Spain), Technovation (vol. 29, n9) pp. 611-617,

51. MENERAULT, PHILIPPE, 1997, Dynamiques et politiques régionales autour du tunnel sous la Manche et du T.G.V. Nord, Annales de géographie (vol. 106, n 5933) pp. 5-33 
52. MVA, 2007, Etude de l'évolution de l'accessibilité des territoires lorrains au réseau TGV Est. Synthèse de l'étude, pour Direction Régionale de l'Equipement de Lorraine, http://www.lorraine.equipement.gouv.fr/IMG/pdf/Synthese_cle5ed71b.pdf

53. NASH, CHRIS A, 1991, The case for high speed rail, Investigaciones economicas (Segunda época) (vol. 15, n²), pp. 337-354

54. NASH, CHRIS, 2009, Quand investir dans des lignes à grande vitesse ? (discussion paperInternational transport research symposium-Madrid 2009), OCDE, FIT, document de référence n²009-16, http://www.internationaltransportforum.org/Proceedings/Symp2009/2Nashfr.pdf

55. OFFNER, JEAN-MARC, 1993, Les "effets structurants" du transport : mythe politique, mystification scientifique, L'espace géographique $n$ ^3, http://www.sciences-pourbanisme.fr/IMG/pdf/article-offner-espace-geo.pdf

56. ORANGe County Business COUNCIL, 2008, The economic impact of high speed trains for Orange County, http://www.cahighspeedrail.ca.gov/images/chsr/20081020174618_HSROCReport.pdf

57. O'TOOLE, RANDAL, 2009, The high cost of high-speed rail, pour Texas Public Policy Foundation, http://www.texaspolicy.com/pdf/2009-08-RR03-HSR-rotoole.pdf

58. PARIS, CHRIS, 1992, The slow death of a Very Fast Train: government resistance to a privately funded transport innovation, International journal of urban and regional research (vol. 16, n4) pp. 623-632

59. PARK, YONGHWA; HA, HUN-KoO, 2006, Analysis of the impact of high-speed railroad service on air transport demand, Transportation research (Part E: logistics and transportation review) (vol. 42, n²) pp. 95-104

60. PETERS, DEIKE, 2009, The renaissance of inner-city rail station areas as a key element in the contemporary dynamics of urban restructuring, Paper for Critical Planning's 2009 Special Issue on Urban Restructuring

61. PLASSARD FRANÇOIS, 2003, Transport et territoire, La Documentation Française, Paris, 97 p.

62. PLASSARD FrançOIS, 1977, Les autoroutes et le développement régional, Presses Universitaires de Lyon

63. POL, Peter M. J, 2003, The economic impact of high-speed train on urban regions, pour European regional science association (conference paper), http://www-sre.wuwien.ac.at/ersa/ersaconfs/ersa03/cdrom/papers/397.pdf

64. PRESTON, JOHN ; LARBIE, AdAM ; WALL, GRAHAM, The impact of high speed trains on socio-economic activity : the case of Ashford (Kent), To be presented to the 4th Annual Conference on Railroad Industry Structure, Competition and Investment, Universidad Carlos III de Madrid, http://163.117.2.172/temp/agenda/mad2006/papers/25.\%20Preston.pdf

65. PRESTON, JOHN, 2009, The case for high speed rail : a review of recent evidence, pour Royal Automobile Club Foundation for Motoring (rapport $n^{\circ}$ 09/128)

66. PUGA, DIEGO, 2001, European regional policies in light of recent location theories, To be published in Journal of economic geography (vol.2, $\mathrm{n}{ }^{\circ}$ )

67. RAVE (REDE FERROVIARIA DE ALTA VELOCIDAD SA), The High-Speed Rail project portugais Communiqué de presse, http://www.rave.pt/en/tabid/389/Default.aspx

68. RFF (REseau FerRe de France), 2010, Bilan de 25 ans de construction de LGV. Les LGV à I'heure du bilan, Lignes d'avenir (n 8 ), http://www.rff.fr/lMG/lignedavenir-fev2010.pdf

69. RIEVELD, PIET; BRUINSMA, FRANCK; VAN DELFT, H. T. ; UBBELS, B., 2001, Economic impacts of high speed trains. Experiences in Japan and France: expectations in The 
Netherlands, Serie Research Memoranda (de : Faculteit der Economische Wetenschappen en Bedrijfskunde), $\mathrm{n}{ }^{\circ 20}$

70. RUS, GINÉS DE; NOMBELA, GuSTAVO, 2007, Is investment in high speed rail socially profitable? , Journal of transport economics and policy (vol. 41, n¹), pp.3-23

71. SANDS, BRIAN D, 1993, The Development Effects of High-Speed Rail Stations and Implications for California, Institute of Urban and Regional Development University of Berkeley, working paper, http://www.uctc.net/papers/115.pdf

72. SETEC ORGANISATION, 2005, Etude des effets de la réalisation d'une LGV entre Bordeaux et Narbonne sur le développement des aires urbaines d'Agen et de Montauban et sur l'aménagement des territoires traversés, pour Réseau Ferré de France,http://cpdp.debatpublic.fr/cpdp-lgv-bordeaux-toulouse/docs/pdf/etudes/ amenagement-\%28synthese\%29.pdf

73. SHEFER, DANIEL; AVIRAM, HAIM, 2005, Incorporating agglomeration economies in transport cost-benefit analysis : the case of the proposed light-rail transit in the Tel-Aviv metropolitan area, Papers in regional science (vol. 84, $\mathrm{n}^{\circ 3}$ ) pp. 487-508

74. SHERWOOD, BOB, 2009, Rail link lifts property value hopes, Financial Times

75. SNCF, 2008, Dossier de presse : TGV et TER fêtent les 1 an de la ligne Est Européenne, http://www.vialibre-ffe.com/PDF/1er_ano_tgv_est.pdf

76. South East England Development Agency (SEEDA), 2008, HST impact study. Final report, pour la Commission européenne,

http://www.hstimpactstudy.net/HTdocs/lmages/mainreport150408.pdf

76. SPAVEN, DAVID, 2006, Are High-speed railways good for the environment ? A discussion paper, pour Transform Scotland,

www.transformscotland.org.uk/GetFile.aspx? Itemld=92

78. STUDER, ELIZABETH, 2008, TGV Lisbonne/Madrid : appel d'offres début juin, http://www.leblogfinance.com/2008/01/tgv-lisbonnemad.html

79. STUDER, ElizABeth, 2008, Portugal : TGV entre Porto et Vigo prévu pour 2013, http://www.leblogfinance.com/2008/04/portugal-tgv-en.html

80. THOMPSON, LOUIS S, 1994, High-speed rail (HSR) in the Unites-States -Why isn't there more? , Japan railway \& transport review, pp. 32-39

81. TRANSPORTATION ECONOMICS \& MANAGEMENT SYSTEMS, 2007, Ohio hub passenger rail economic impact study. Chapter 7 : economic rent analysis and results, http://www.beatthejam.org/ohiorail/Ohio\%20Hub/Website/ordc/Economic_Impact_Study/7_E conomic_Rent_Analysis_and_Results.pdf

82. TRANSPORTATION ECONOMICS \& MANAGEMENT SYSTEMS, 2007, Ohio hub passenger rail economic impact study. Chapter 12 : tourism impacts, http://www.dot.state.oh.us/Divisions/Rail/Programs/passenger/Ohio\%20Hub\%20Economic\% 20 Impact\%20Studies/TEMS12Tourismlmpacts.pdf

83. TROIN, JEAN-FRANÇOIS, 1997, Les gares T.G.V. et le territoire : débat et enjeux, Annales de géographie (vol. 106, n ${ }^{\circ 593)}$ pp. 34-50

84. TROIN, JEAN-FRANÇOIS ; Fédération Nationale des Associations d'Usagers des Transports (FNAUT), 2008, Les gares nouvelles du TGV "exurbanisées". Fonctionnement et relation au territoire, pour la DIACT, FNAUT, Paris.

http://temis.documentation.equipement.gouv.fr/documents/Temis/0063/Temis-

0063302/17669.pdf 
85. URENA, José M; MENERAULT, PHILIPPE; GARMENDIA, MADDI, 2009, The high-speed rail challenge for big intermediate cities : a national, regional and local perspective, Cities (vol. 26, n ०5) pp. 266-279

86. VICKERMAN R.W., 1991, Infrastructure and regional development, Pion Limited, London

87. VICKERMAN R.W., 1997, High-speed rail in Europe experience and issues for future development, Annals of Regional Science, 31, pp. 21-38

88. VICKERMAN, ROGER; ULIED, ANDREU, 2006, Indirect and wider economic impacts of highspeed rail,

http://163.117.2.172/temp/agenda/mad2006/papers/12.\%20Vickerman,\%20Roger.pdf

89. VilLes et REGIONS eUROPEenNeS DE LA GRANDE ViteSSE ; Reims Métropole, 2006, Grande Vitesse ferroviaire : quelles retombées pour les collectivités locales ? Synthèse du colloque,http://pagesperso-orange.fr/asso-villestgv/documents/colloque 08-0306_synthese.pdf

90. WILLIGERS, JASPER; FLOOR, HAN; VAN WEE, BERT, 2005, High-speed rail's impact on the location of office employment within the Dutch Randstad Area, 45th Congress of the European Regional Science Association, Amsterdam, August 23-27, 2005, http://wwwsre.wu-wien.ac.at/ersa/ersaconfs/ersa05/papers/308.pdf

91. WOLKOWITSCH, MAURICE, 1987, Le développement du réseau mondial des lignes à grande vitesse : réalisations et projets, Annales de géographie (vol. 96, n 535) pp. 273-291

92. ZEMBRI, PieRRE, 1993, T.G.V. - Réseau ferré classique : des rendez-vous manqués ?, Annales de géographie (vol. 102, n ^571) pp. 282-295 